\title{
ATIVIDADES PARA O CONTROLE DA TUBERCULOSE NA ATENÇÃO PRIMÁRIA À SAÚDE DE UM MUNICÍPIO BRASILEIRO
}

\section{ACTIVITIES FOR THE CONTROL OF TUBERCULOSIS IN PRIMARY HEALTH CARE OF A BRAZILIAN CITY}
Vitória Daeny Medeiros do Nascimento ${ }^{1}$ Sandy Yasmine Bezerra e Silva ${ }^{2}$
Vitória Keller Gregorio de Araújo ${ }^{3}$ Rayane Saraiva Felix ${ }^{4}$
Rayla Patrícia da Silva Andrade Soares ${ }^{5}$ Érika Simone Galvão Pinto ${ }^{6}$

RESUMO: OBJETIVO: Descrever as atividades desenvolvidas no Programa de Controle da Tuberculose no município de Natal, RN. MÉTODOS: Estudo descritivo, de abordagem quantitativa, realizado no município de Natal, capital do estado do Rio Grande do Norte. O estudo contou com a participação de 80 enfermeiros e a coleta de dados foi realizada no período de novembro de 2017 a fevereiro de 2018, utilizando um questionário elaborado pelos pesquisadores. Os dados foram organizados e analisados através do programa Statistical Package for the Social Science. RESULTADOS: Entre as atividades desenvolvidas no controle da tuberculose do município destaca- se positivamente a orientação para coleta de escarro (90\%), a solicitação do raio-X (60\%), testagem anti-HIV (91,2\%). Como atividades menos desenvolvidas identificou-se a solicitação do teste rápido molecular para o diagnóstico da Tuberculose (45\%), a oferta de Tratamento Diretamente Observado (47,5\%) e a busca ativa de Sintomático Respiratório (45\%). CONCLUSÃO: Foram identificados pontos positivos e negativos na operacionalização das atividades de controle da doença, e esses achados podem

\footnotetext{
1 Graduanda do curso de Enfermagem da Universidade Federal do Rio Grande do Norte.

2 Mestre em Enfermagem pelo Programa de Pós Graduação em Enfermagem da Universidade Federal do Rio Grande do Norte.

${ }^{3}$ Graduanda do curso de Enfermagem da Universidade Federal do Rio Grande do Norte.

${ }^{4}$ Enfermeira Mestranda pelo Programa de Pós Graduação em Enfermagem da Universidade Federal do Rio Grande do Norte.

${ }^{5}$ Enfermeira Mestranda pelo Programa de Pós Graduação em Enfermagem da Universidade Federal do Rio Grande do Norte.

6 Doutora em Enfermagem em Saúde Pública. Professora do Departamento de Enfermagem da Universidade Federal do Rio Grande do Norte.
} 
contribuir com as equipes de saúde na assistência, bem como ferramenta para os gestores locais organizarem sua rede.

Descritores: Tuberculose; Atenção Primária à Saúde; Enfermagem.

ABSTRACT: OBJECTIVE: Describe the activities developed in the Tuberculosis Control Program in the city of Natal, RN. METHOD: A descriptive study with a quantitative approach, conducted in Natal, capital of the state of Rio Grande do Norte. The study was attended by 80 nurses and data collection was performed from November 2017 to February 2018, using a questionnaire prepared by the researchers. Data were organized and analyzed using the Statistical Package for Social Science program. RESULTS: Among the activities carried out in the control of tuberculosis in the city, we highlight the orientation for sputum collection (90\%), X-ray request (60\%), anti-HIV testing (91.2\%). Less developed activities were the request for the molecular rapid test for the diagnosis of tuberculosis (45\%), the offer of directly observed treatment (47.5\%) and the active search for respiratory symptoms (45\%). CONCLUSION: Positive and negative points were identified in the operationalization of disease control activities, and these findings may contribute to health care teams, as well as a tool for local managers to organize their network.

Descriptors: Tuberculosis; Primary Health Care; Nursing. 


\section{INTRODUÇÃO}

A tuberculose (TB) é a doença infectocontagiosa que mais mata em todo o mundo e configura-se como um grande problema de saúde pública com forte vertente social, como a baixa renda e vulnerabilidade. São estimados 10 milhões de casos em 2018, segundo a Organização Mundial de Saúde (OMS, 2019). O Brasil é um dos países com maior incidência de TB, chegando a 72.788 novos casos em 2018 (BRASIL, 2019).

Dada a proporção da doença no Brasil, o Programa Nacional de Controle da Tuberculose (PNCT) é responsável por estabelecer diretrizes e estruturar, dentro do Sistema Único de Saúde (SUS), os serviços e a assistência, envolvendo as 3 esferas nacionais de governo - municipal, estadual e federal - quanto às ações para o controle e redução da TB, com foco na busca ativa de sintomáticos respiratórios (SR), diagnóstico e prevenção de casos (CECÍLIO et al, 2017).

Apesar das diretrizes serem padronizadas, os programas de controle da doença a nível municipal são operacionalizados de formas distintas e geram resultados diferenciados (ARAKAWA et al, 2017).

Estudo realizado no cenário amazônico evidenciou lacunas na operacionalização das atividades de controle da doença relacionadas aos sistemas de informação em saúde, como a dificuldade no planejamento, execução das metas de controle; à realização da cultura e prova tuberculínica (HEUFMAN; GONÇALVES; GARNELO, 2013).

Em relação ao município de Natal, segundo Coelho (2014), dificuldades relacionadas à realização de exames diagnósticos foram evidenciadas, o que pode comprometer o controle da doença.

É imprescindível conhecer a operacionalização atual do programa de controle no município, de modo a relacionar os achados ao processo de trabalho das equipes de saúde e dos gestores, para a sua possível implementação. 
Assim, o objetivo do estudo é descrever as atividades desenvolvidas no Programa de Controle da Tuberculose (PCT) no município de Natal, RN.

\section{MÉTODOS}

Trata-se de um estudo descritivo, de abordagem quantitativa. A presente pesquisa faz parte do projeto "Avaliação do Grau de Implantação do Programa de Controle da Tuberculose no município de Natal/RN".

O estudo foi realizado no município de Natal, capital do estado do Rio Grande do Norte, que possui 54 Unidades de Saúde (US) na APS e contou com a participação de 80 enfermeiros. Os critérios de inclusão foram: Enfermeiros que estivessem em atividade no serviço durante o período da coleta e que desempenhassem atividades no controle da TB. Foram excluídos aqueles que desempenhassem ações no controle da TB que não estivessem lotados na APS.

A coleta de dados foi realizada no período de novembro de 2017 a fevereiro de 2018, utilizando um questionário elaborado pelos pesquisadores com base no Manual de Recomendações para o Controle da Tuberculose no Brasil (BRASIL, 2011), composto por 62 questões semiestruturadas. Para o presente estudo foram utilizadas 12 questões que abordavam as atividades do PCT.

As variáveis utilizadas no questionário acerca das atividades realizadas no programa foram subdivididas em operacionais e às relacionadas aos sistemas de informação, estão apresentadas no quadro 1. 
Quadro 1 - Variáveis utilizadas no questionário em relação às atividades do Programa de Controle da Tuberculose em Natal, RN - Natal - 2017.

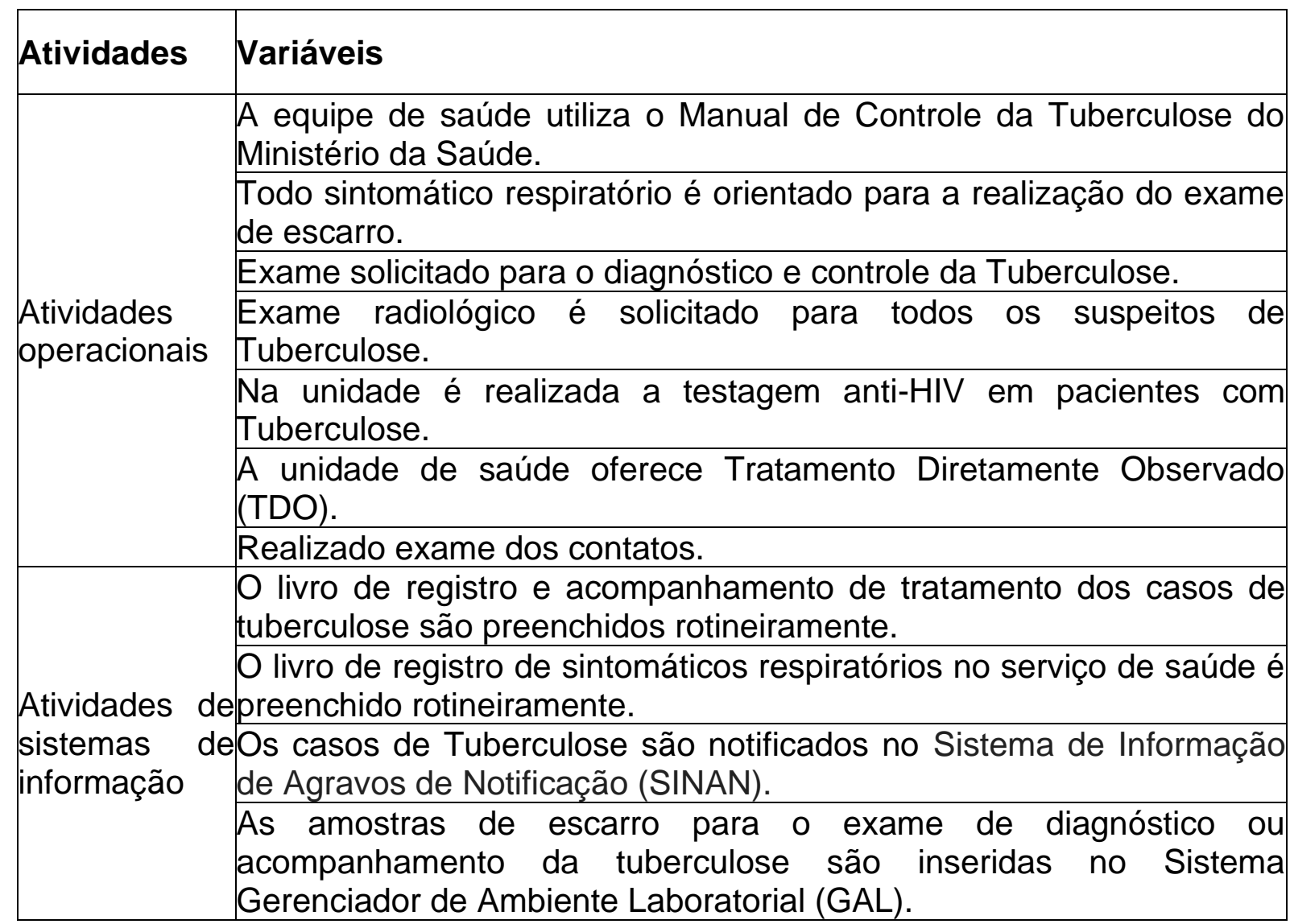

Fonte: Produção própria (2019).

Os dados foram organizados e analisados através do programa Statistical Package for the Social Sciences (SPSS), versão 22.0 IBM, utilizando-se estatística descritiva em números absolutos e relativos.

A pesquisa possui aprovação do Comitê de Ética em Pesquisa da UFRN, parecer n. 2.327.693 e foram respeitadas as disposições da Resolução 466/2012.

\section{RESULTADOS}

Participaram do estudo 80 enfermeiros, dos quais a maioria era do sexo feminino $(93,7 \%)$, com idade média de 48 anos e tempo médio de atuação nesta 
função, 18 anos. Destes, 92,5\% afirmou ter recebido capacitação em TB. Na tabela 1 estão apresentadas as atividades operacionais desenvolvidas pelos participantes da pesquisa:

Tabela 1 - Atividades operacionais desenvolvidas no Programa de Controle da Tuberculose no unicípio de Natal, RN, Brasil, 2018.

Atividades operacionais

Frequência (n) Percentual (\%)

A equipe de saúde utiliza as normas do Ministério da Saúde (MS) para o diagnóstico e tratamento da pessoa com TB

$\begin{array}{ccc}\text { Quase nunca } & 01 & 1,2 \\ \text { Às vezes } & 02 & 2,5 \\ \text { Quase sempre } & 05 & 6,3 \\ \text { Sempre } & 72 & 90,0 \\ \text { Total } & 80 & 100,0\end{array}$

Todo sintomático respiratório é orientado para a realização do exame de escarro

$\begin{array}{ccc}\text { Quase nunca } & 00 & 0,0 \\ \text { Às vezes } & 04 & 5,0 \\ \text { Quase sempre } & 04 & 5,0 \\ \text { Sempre } & 72 & 90,0 \\ \text { Total } & 80 & 100,0\end{array}$

Exame solicitado para o diagnóstico e controle da TB

$\begin{array}{ccc}\text { Teste rápido molecular (TRM-TB) } & 36 & 45,0 \\ \text { Baciloscopia } & 76 & 95,0 \\ \text { Cultura + Teste de sensibilidade } & 22 & 27,5 \\ \text { Outro } & 09 & 11,3 \\ \text { O exame radiológico é solicitado para todos os suspeitos de TB } \\ \text { Sim } & 48 & 60,0 \\ \text { Não } & 32 & 40,0 \\ \text { Total } & 80 & 100,0\end{array}$

A sua unidade realiza a testagem anti-HIV em pacientes com TB

$\begin{array}{ccc}\text { Quase nunca } & 00 & 0,0 \\ \text { Às vezes } & 01 & 1,3 \\ \text { Quase sempre } & 06 & 7,5 \\ \text { Sempre } & 73 & 91,2 \\ \text { Total } & 80 & 100,0 \\ & \text { É oferecido TDO na sua unidade } \\ \text { Sim } & 38 & \\ \text { Não } & 39 & 47,5 \\ \text { Não respondeu } & 03 & 48,7 \\ \text { Total } & 80 & 3,8 \\ \text { Total } & \end{array}$


Você realiza busca de sintomáticos respiratórios

Nunca 11

Quase nunca

Às vezes

Quase sempre

Sempre

Não respondeu

Total
03

18

11

36

01

80
13,7

3,8

22,6

13,7

45,0

1,2

100,0

Você realiza exame dos contatos

$\begin{array}{ccc}\text { Nunca } & 03 & 3,7 \\ \text { Quase nunca } & 02 & 2,5 \\ \text { Às vezes } & 05 & 6,3 \\ \text { Quase sempre } & 13 & 16,3 \\ \text { Sempre } & 57 & 71,2 \\ \text { Total } & 80 & 100,0\end{array}$

Entre as atividades operacionais desenvolvidas no PCT do município destacase positivamente a orientação para coleta de escarro (90\%), a solicitação do raio-x (60\%) e testagem anti-HIV (91,2\%). Como atividades menos desenvolvidas destacase a solicitação do teste rápido molecular para o diagnóstico da TB (45\%), a oferta de TDO (47,5\%) e a busca ativa de SR (45\%). Na tabela 2 estão apresentadas as atividades relacionadas aos sistemas de informação em saúde:

Tabela 2 - Atividades relacionadas aos sistemas de informação em saúde desenvolvidas no Programa de Controle da Tuberculose no município de Natal, RN, Brasil, 2018.

\section{Atividades de sistema de saúde de informação Frequência (n) Percentual (\%)}

O livro de registros e acompanhamento de tratamento dos casos de tuberculose é preenchido rotineiramente

$\begin{array}{ccc}\text { Nunca } & 00 & 0,0 \\ \text { Quase nunca } & 01 & 1,2 \\ \text { Às vezes } & 04 & 5,0 \\ \text { Quase sempre } & 10 & 12,5 \\ \text { Sempre } & 64 & 80,0 \\ \text { Não respondeu } & 01 & 1,2 \\ \text { Total } & 80 & 100,0\end{array}$

O livro de registros de sintomáticos respiratórios no serviço de saúde é preenchido rotineiramente 


$\begin{array}{ccc}\text { Nunca } & 04 & 5,0 \\ \text { Quase nunca } & 03 & 3,8 \\ \text { Às vezes } & 07 & 8,7 \\ \text { Quase sempre } & 13 & 16,3 \\ \text { Sempre } & 52 & 65,0 \\ \text { Não respondeu } & 01 & 1,2 \\ \text { Total } & 80 & 100,0 \\ \text { Os casos de TB são notificados no SINAN } & \\ \text { Nunca } & 01 & 1,3 \\ \text { Quase nunca } & 01 & 1,3 \\ \text { Às vezes } & 01 & 1,2 \\ \text { Quase sempre } & 00 & 0,0 \\ \text { Sempre } & 76 & 95,0 \\ \text { Não respondeu } & 01 & 1,2 \\ \text { Total } & 80 & \end{array}$

As amostras de escarro para exame diagnóstico ou de acompanhamento da tuberculose são inseridas no GAL

\begin{tabular}{ccc} 
Nunca & 08 & 10,0 \\
Quase nunca & 01 & 1,2 \\
Às vezes & 03 & 3,8 \\
Quase sempre & 01 & 1,3 \\
Sempre & 66 & 82,5 \\
Não respondeu & 01 & 1,2 \\
Total & 80 & 100,0 \\
\hline
\end{tabular}

Diante dos dados pode-se identificar que as atividades relacionadas aos sistemas de informação em saúde são realizadas em quase a sua totalidade no município.

\section{DISCUSSÃO}

No presente estudo, a orientação para a coleta de exame de escarro foi uma atividade identificada como sempre executada. As orientações são importantes para melhora da qualidade da amostra para o exame e levam a maior interação entre usuário e profissional. Também estão relacionadas à diminuição do tempo entre o exame diagnóstico e a confirmação do caso, diminuindo a propagação da doença (SICSÚ et al, 2016). 
A solicitação de raio-x em caso suspeito de TB também foi identificada como desenvolvida. O exame radiológico é um ponto importante para o diagnóstico precoce, mas estudo realizado por Ponce (2016) constatou que essa etapa pode ficar comprometida por deficiências e fragilidades dos profissionais da assistência quanto aos conhecimentos e capacitações para manejo da doença.

Quanto à testagem anti-HIV, constituiu-se como uma atividade sempre desempenhada pela equipe do programa de controle da TB. Magno (2017) aborda sobre o risco de pessoas infectadas pelo HIV terem chances aumentadas em cerca de $20 x$ de adquirirem TB tanto por reativação de infecção latente pelo Mycobacterium tuberculosis como por reinfecção. Os testes rápidos são uma estratégia fundamental para o controle da doença, tendo em vista a rapidez em que é possível visualizar o resultado e o custo benefício para executar o teste (BRASIL, 2019).

Dentre os exames solicitados para o diagnóstico e controle da TB, identificouse que o TRM-TB, utilizado para a detecção de DNA dos bacilos do complexo $M$. tuberculosis e triagem de cepas resistentes à rifampicina, é menos solicitado para diagnóstico em relação à baciloscopia o município em questão. O Manual de Recomendações para o controle da TB no Brasil (2019) preconiza que o TRM-TB seja solicitado preferencialmente para o diagnóstico de TB pulmonar e laríngea em adultos e adolescentes. Esse dado pode implicar em atraso nos diagnósticos, comprometendo o controle da doença.

Com relação à oferta de TDO, identificou-se que não é oferecido em parte das unidades de saúde, dado preocupante, visto que o TDO contribui para o controle da doença e está associado à adesão ao esquema terapêutico. Verifica-se ainda, como uma ferramenta importante para 0 acolhimento e fortalecimento do vínculo profissional/usuário no que tange ao desenvolvimento das ações voltadas para a TB (CECILIO; MARCON, 2016).

Quanto à busca ativa de SR foi obtido um resultado positivo dos profissionais na qual, constaram que sempre realizam a busca em sua área adscrita. Para Gabardo et al. (2019), essa é uma atividade importante para o diagnóstico precoce da TB, desenvolvida como uma estratégia de controle e interrupção da cadeia de transmissão da doença. 
Em relação ao registro de informações, os resultados apontaram que tanto o livro de acompanhamento de tratamento dos casos de TB quanto o livro de registro de SR são preenchidos rotineiramente. Estes resultados corroboram com os encontrados por Barros (2017), cujo item registro sobre doentes alcançou ótima capacidade em relação aos indicadores de desempenho no acompanhamento de pessoas portadora de tuberculose no município do Rio de Janeiro.

No que se referem à notificação dos casos de TB no Sistema Nacional de Agravos de Notificação (SINAN) os entrevistados relataram realizar sempre. Este resultado corrobora com estudo realizado por Rêgo et al. (2015) no qual foi ressaltado que com o preenchimento das fichas de notificação e investigação é garantido o acesso aos casos por parte dos órgãos responsáveis, e isto possibilita o aumento dos investimentos e da assistência ao paciente na APS.

No que diz respeito à inserção das informações no GAL sobre as amostras de escarro para exame diagnóstico da TB foi identificada como sempre realizada, sendo este resultado superior à média nacional de acordo com o estudo de Clementino et al. (2016) cujo registro mostrou-se presente em 51\% das unidades básicas de saúde.

Os dados referentes aos sistemas de informação à saúde do presente estudo sugerem que essas atividades são prioridades para os profissionais do município em questão.

Elenca-se como limitação deste estudo o fato da pesquisa não ter envolvido a totalidade de profissionais da rede de serviços de APS do município.

\section{CONCLUSÕES}

Nesse estudo foi possível descrever as atividades desenvolvidas no Programa de Controle da Tuberculose no município de Natal, RN, com destaque para a orientação para coleta de escarro, a solicitação do raio-x, testagem anti-HIV e às relacionadas aos sistemas de informação em saúde. 
Em contrapartida, algumas fragilidades merecem atenção especial, principalmente referente à solicitação do teste rápido molecular para o diagnóstico da TB, a oferta de TDO e a busca ativa de SR. Essses achados podem contribuir com as equipes de saúde da APS no planejamento do cuidado, bem como ferramenta para os gestores locais organizarem sua rede, no intuito de garantir à pessoa com TB um cuidado integral.

\section{REFERÊNCIAS BIBLIOGRÁFICAS}

ARAKAWA, T.; MAGNABOSCO, G. T.; ANDRADE, R. L. P.; BRUNELLO, M. E. F.; MONROE, A. P.; RUFFINO-NETTO, A. Tuberculosis control program in the municipal context: performance evaluation. Rev. Saúde Pública, São Paulo, v. 51, n. 23. 2017.

BARROS, R. S. L. A Estratégia Saúde da Família e o Programa de Controle da Tuberculose no Município do Rio de Janeiro. Tese. Universidade Federal do Rio de Janeiro. 2017.

BRASIL. Ministério da saúde. Secretaria de Vigilância em saúde. Departamento de Vigilância das doenças transmissíveis. Manual de Recomendações para o controle da tuberculose no Brasil. Brasília: Ministério da saúde, 2011.

BRASIL. Ministério da saúde. Secretaria de Vigilância em saúde. Departamento de Vigilância das doenças transmissíveis. Manual de Recomendações para o controle da tuberculose no Brasil. Brasília: Ministério da saúde, 2019.

BRASIL. Ministério da Saúde. Secretaria de Vigilância em Saúde. Plano Nacional pelo Fim da Tuberculose. Boletim Epidemiológico, Brasília, v. 50, n. 09, 18 p. Mar. 2019.

CECILIO, H. P. M.; MARCON, S. S. O tratamento diretamente observado da tuberculose na opinião de profissionais de saúde. Revista Enfermagem UERJ, v. 24, n. 1, n. esp. e8425, jul. 2016.

CECILIO, H. P. M.; TESTON, E. F.; MARCON, S. S. Acesso ao diagnóstico de tuberculose sob a ótica dos profissionais de saúde. Texto contexto - Enferm. Florianópolis, v. 26, n. 3, 2017.

CLEMENTINO, F. S., MARCOLINO, E. C., GOMES, L. B., GUERREIRO, J. V., MIRANDA, F. A. N. Ações de controle da tuberculose: Análise a partir do Programa de Melhoria do Acesso e da Qualidade da Atenção Básica. Texto Contexto Enferm, 2016; v. 25, n. 4, p. 1-9.

COELHO, A. A.; MARTINIANO, C. S.; BRITO, E. W. G.; NEGRÃO, O. G. C.; ARCÊNDIO, R. A.; UCHÔA, S. A. C. Tuberculosis care: an evaluability study. Revista Latino- Americana de Enfermagem. Ribeirão Preto, v. 22, n.5, p. 792-800, 2014.

GABARDO, B. M. A. MALUF, E. M. C. P.; FREITAS, M. B. F.; GABARDO, B. A. É necessário realizar busca ativa de sintomáticos respiratórios independentemente dos cenários epidemiológicos locais? J. bras. pneumol. São Paulo, v. 45, n. 6, n. esp. e20190171, 2019.

HEUFEMANN, N. E. C.; GONÇALVES, M. J. F.; GARNELO, M. L. Avaliação do programa de 
controle da tuberculose em cenário Amazônico: desafios em Coari. Acta Amazônica, v. 43, n. 1, p.33-42. 2013.

MAGNO, E. S.; SARACENI, V.; SOUZA, A. B.; MAGNO, R. S.; SARAIVA, M. G. G.; BÜHRERSÉKULA, S. Fatores associados à coinfecção tuberculose e HIV: o que apontam os dados de notificação do Estado do Amazonas, Brasil, 2001-2012. Cad. Saúde Pública, Rio de Janeiro, v. 33, n. 5, 12 jun. 2017.

PONCE, M. A. Z. WYSOCKI, A. D.; ARAKAWA, T.; ANDRADE, R. L. P.; VENDRAMINI, S. H. F.; SOBRINHO, R. A. S.; MONROE, A. A.; NETTO, A. R.; VILLA, T. C. S.. Atraso do diagnóstico da tuberculose em adultos em um município paulista em 2009: estudo transversal. Epidemiol. Serv. Saúde, Brasília. Jul-Set 2016.

REGO, C. C. P.; MACEDO, S. M.; ANDRADE, C. R. B.; MAIA, V. F.; PINTO, J. T. J. M.; PINTO, E. S. G. Processo de trabalho da enfermeira junto à Pessoa com tuberculose na atenção primária à saúde. Revista Baiana de Enfermagem, Salvador, v. 29, n. 3, p. 218-228, Jul./Set. 2015.

SICSÚ, A. N.; SALEM, J. I.; FUJIMOTO, L. B. M.; GONZALES, R. I. C.; CARDOSO, M. S. L.; PALHA, P. F. Intervenção educativa para a coleta de escarro da tuberculose: um estudo quase experimental. Rev. Latino-Am. Enfermagem, v. 24, n. esp. e2703, 2016. 